\section{EM Site A.C. Magnetic Field Sources, Surveys and Solutions Part IV: Survey Data Analysis}

Curt Dunnam, Linear Research Associates

Up to the present waypoint in this series on EM site magnetic fields, we have identified typical sources of time-varying magnetic field intensities, examined salient field characteristics and illustrated correct survey methods. Our goal this month is to analyze data collected at a proposed site and answer the key question of whether or not the candidate site is, as far as magnetic fields go, acceptable for EM use. In the process of analyzing the magnetic field survey data we will define some of the interpretive techniques involved and observe the distinction between localized (a.c. power) and non-localized (geomagnetic) time-varying fields. Finally, we will discuss the implications of EM susceptibility threshold vs. measured field ratios when considering remedial site shielding.

Unambiguous, accurate field survey data is required as a basis for EM site acceptability if analysis of that data is to be the final arbiter of whether a site can be "fixed" or must be abandoned. Interfering magnetic fields which fall below well-defined levels, for example, can often be adequately reduced by shielding the site. Since the alternative option of relocating a proposed (or operating) site may be costly in terms of physical, financial and political tradeoffs, it is obviously important to correctly analyze the magnetic field situation before a final decision is made.

Even at relatively low intensities, a.c. magnetic fields (and slower field variations, in the case of elevator or vehicular geomagnetic modulations) can interfere in subtle ways with EM operations, particularly at sites employing FEG, high resolution and/or low beam energy equipment. In the previous article of this series (Part III, "Survey

\section{COSTEFPECTIVE RM MAGNIETIC SHUILDING}

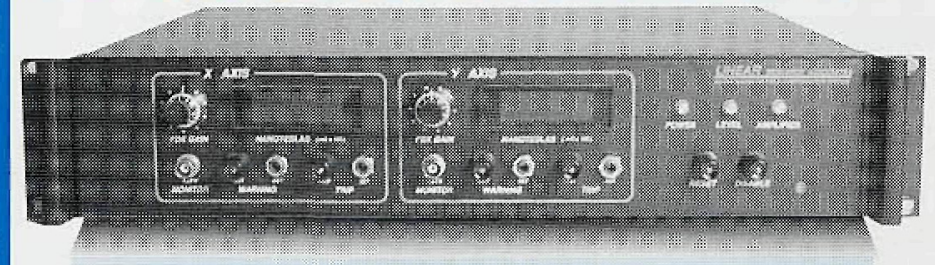

EM sites often require supplemental magnetic shielding to achieve full resolution. LINEAR RESEARCH ASSOCIATES' wideband EMFCAC electronic active-shielding systems dramatically reduce magnetic fields radiating from nearby a.c. power wiring, ground loops, transformers and related sources. EMFC-QDC extended-range models additionally suppress low-frequency magnetic disturbances caused by trains, subways, elevators and equipment such as MRI scanners.

EMFC active-shielding is highly recommended for cost-effective protection of sensitive FEG and PEELS installations.

Linear Research Associates' EMFC equipment is the highestperformance commercially available magnetic field compensation apparatus in the world. EMFC systems feature state-of-the-art engineeringtt and are U.S.-manufactured to exacting standards. Superior performance and reliability are guaranteed!

Call or fax LRA for complete EMFC-series information. We will also gladly assist with any questions you may have regarding site survey, engineering or EMF issues.

\footnotetext{
LINEAR RESEARCH ASSOCIATES

5244 Perry City Road - Trumansburg, NY 14886

Phone (607) 387-3411 • Fax (607) 387-7806

tt U.S. Patents $5,465,012 ; 5,469,058$
}

Instrumentation and Methods", Microscopy Today, January/February "96) we reviewed survey data collecting instrumentation and methodology, and there made a careful distinction between magnetic fields related to a.c. power usage as being quite distinct from "quasi-d.c." fields attributable to vehicles moving through the earth's relatively static field. In the following example data set analyses we will accordingly analyze these two field categories separately.

Presuming an urban, large-building setting, we find ourselves armed with two sets of data magnetic field survey work performed in Part III of this series. Let us first consider the "a.c. magnetic field" [ACMF] survey data, Table 1, which is comprised of powerline-related magnetic field

$\begin{array}{clllll}\text { CELL } & \mathrm{B}_{\mathrm{X}} & \mathrm{B}_{\mathrm{y}} & \mathrm{B}_{\mathrm{z}} & \mathrm{B}_{\mathrm{xY}(\text { meas) }} & \mathrm{B}_{\text {Xz(calc) }} \\ \mathrm{A} & 93.3 & 28.3 & 178 & 95.0 & 204 \\ \mathrm{~B} & 113 & 53.7 & 122 & 116 & 175 \\ \mathrm{C} & 122 & 31.3 & 181 & 124 & 221 \\ \mathrm{D} & 139 & 59.4 & 130 & 153 & 201 \\ \text { E } & 184 & 50.9 & 164 & 195 & 252 \\ \text { F } & 158 & 56.6 & 139 & 161 & 218 \\ \text { @ COLUMN } & 124 & 33.9 & 158 & 130 & 204\end{array}$

Table 1. ACMF fluxgate survey data (values in nT, peak-to-peak, at height=1.5m)

values recorded at several points throughout the site. The room is rectangular with a bird's-eye aspect of approximately 3 to 2 and is divided into roughly square cells which are consecutively lettered $A, B, C$ and $D, E, F$ proceeding from left to right and top to bottom. As noted in the previous article, the tabulated values are recorded with the aid of a calibrated tri-axial fluxgate probe and have been verified by comparison with values observed on a hand-held teslameter.

ACMF's may be characterized in two ways. First, they possess interference components which range from $16 \mathrm{~Hz}$ (e.g., Scandinavian electified trains) to $720 \mathrm{~Hz}\left(12^{\text {th }}\right.$ harmonic of $\left.60 \mathrm{~Hz}\right)$. Also, significant third-harmonic magnetic field energy at $150 / 180 \mathrm{~Hz}$ is quite common. The second important characteristic of ACMF's is that they are frequently produced by local sources such as transformers, motors and video display monitors. Often, transformers and motors are hidden by walls and/or partitions and are only easily "seen" with the hand-held teslameter. Analysis of "hot spot" data noted during a sweep will indicate if a source is contributing in a significant way to magnetic fields at the proposed EM column location. If so, a marginal site may be salvaged by relocating the offending electrical apparatus further away from the EM site.

In the hypothetical room represented by the above table, peripheral equipment imposes strict limitations on instrument placement - and we can locate the EM column only where the corners of cells $A, B, C$ and $D$, or cells $C$, $D, E$ and $F$ touch. Let us assume initially that our EM is a standard non-FEG instrument, and exhibits a typical susceptibility threshold of $250 \mathrm{nTp}-\mathrm{p}$ [2.5 $m G p-p]$ in the $X Y$ plane and $500+n T p-p[5.0 \mathrm{mGp}-\mathrm{p}]$ in the $Z$ (vertical) axis. Reviewing the above table figures, we observe that we are on fairly safe ground as far as the environmental ACMF fields are concerned. If, on the other hand, we are expecting to operate a FEG-equipped or high-resolution or low eV instrument in that room, some type of shielding will be necessary. Under the $\mathrm{B}_{\mathrm{x} 2}$ conditions listed in the final column of Table 1, and with a typical $150 \mathrm{nTp} p \mathrm{p}$ [1.5 mGp-p] isotropic interference threshold, such instruments would probably not deliver full resolution specs.

Next, we look at the "quasi-d.c." [QDC] data which we prudently collected (having previously noted that our building is full of exotic equipment and a subway line runs beneath it). As described in our last installment, the initial survey step for QDC fields is to fix a probe near the center of the EM room and manually note all axial field variations occurring in a frequency range of 0 to 1.6 $\mathrm{Hz}$ over a period of two minutes or more. That data is tabulated (Table 2) and immediately analyzed

$\begin{array}{lrrrr}\text { AXIS } & \text { LOW } & \text { HIGH } & \Delta_{1} \text { ABS } & \Delta_{1} \text { REL, \% } \\ & \text { UTp-p } & \text { UTp-p } & \text { UTp-p } & \\ X & 11.08 & 11.35 & 0.27 & 2.4 \\ \text { Y } & 2.13 & 274 & 0.61 & 2.2 \\ Z & 15.30 & 16.20 & 0.90 & 5.6\end{array}$

Table 2. Site "QDC" short-term (2 minute) sample data. 


\section{AEW PRODUOT AEWS}

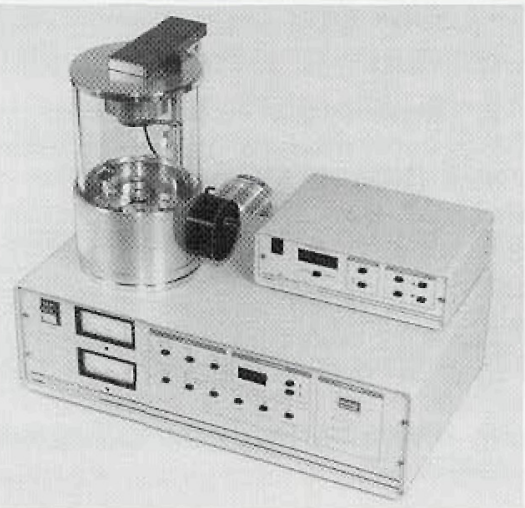

\section{HIGH RESOLUTION SPUTTER COATER FOR FE-SEM}

This high resolution sputter coater is equipped with both Chromium and Platinum/Palladium targets, rotary and turbo drag pump combination, and separate rotary, planetary and tilting sample stage movements as standard. Thickness optimized to the FE-SEM operating voltage using the high resolution thickness controller
Benchtop design saves space and energy. Contact Ted Pella, Inc

P.O. Box 492477, Redding CA 96049-2477 (916)243-2200 - Fax: $(916) 243-3761$

Circle Reader Inquiry \#40
Philips Electron Optics Introduces the XL50: a new Defect Review Tool of the Semiconductor Industry

The XL50 is a highly integrated tool that combines an easy user interface, proven analytical performance and exceptional stage accuracy Intended for use in the low yield analysis lab, the XL50 will allow classification of defects with imaging resolution of 3-5 $\mathrm{nm}$. This data can then be fed back into quality control and continuous improvement processes, helping to minimize manufacturing deviations and hence raise yields and plant profitability

The XL50 combines a field emission electron source with a specially developed $200 \times 200 \mathrm{~mm}$ 5 -axis movement stage. The stage average accuracy is within 1.5 microns across an entire 8 wafer (or 0.6 microns over a $25 \mathrm{~mm}$ die). Menu driven operation entirely under the familiar MS-Windows environment means operators can classify defects in a user-friendly manner. The high precision stage enables a wide variety of wafers and wafer parts to be examined and navigation is both intuitive and straight-forward. Circle Reader Inquiry \#42

\section{Micro Plane}

Sample Preparation Tool for Microscopy

The Micro Plane tools from Spectra-Tech Inc are useful sample preparation tools for any microscopy laboratory, especially one that employs infrared microscopy. Samples prepared for infrared microanalysis need to meet specific criteria for transmission analyses where the ideal sample thickness is between 5 and 20 microns. This device allows the user to plane or scrape the surface of polymers or other solid materials to produce a uniform slice thickness. Thickness is controlled through simple adjustment of the angle and position of

\section{the Micro Plane's blade}

The Micro Plane is available in two configurations having either a carbide-steel blade version or a diamond-edge blade. For further information, contaci Debbie Esposito at ( 800$) 243-9186$ Circle Reader Inquiry \#41 to determine which two axes are to be monitored for a total period of at least 24 hours using a dual-channel chart recorder (ref. Part III). In this case, we have chosen axes $Y$ and $Z$. With our completed chart (or charts) in hand, the statistics and magnitude of peak-to-peak low-frequency magnetic field variations occurring within any eight minute window (i.e., corresponding to -20 $\mathrm{dB}$ with respect to the lowest frequency of interest) are carefully noted. Let us assume here that the relevant peak-to peak QDC variations discerned on the chart are $1.5 \mu \mathrm{Tp}-\mathrm{p}$ [15 mGp-p] in the $Y$ axis and $2.5 \mu \mathrm{Tp}-\mathrm{p}$ [25 mGp-p] in the $Z$ axis. It is readily apparent that these variations are over an order of magnitude greater than the ACMF EM threshold specs. Worse still, the EM conductive shrouds and UHV containment are relatively ineffective in blocking QDC field variations below $16 \mathrm{~Hz}$, so the EM's exhibit up to $30 \%$ more sensitivity to magnetic field variations in this frequency range. All factors considered, our measurements indicate field modulations in the room are approximately 24 times greater than the interference threshold for a FEG instrument! From the standpoint of probable magnetic field interference, this site is clearly unacceptable in its present state for any of the previously mentioned instrument classes.

Nonetheless, since the observed magnetic field variation to $\mathrm{EM}$ susceptibility ratio is less than 25 , the site may in fact be usable if magnetic shielding is employed. We will discuss that encouraging prospect in the next article of this series.

Questions and/or comments relating to this series are welcomed and may be faxed to the author's attention at Linear Research Associates, Trumansburg, NY (USA) 607-387-7806.

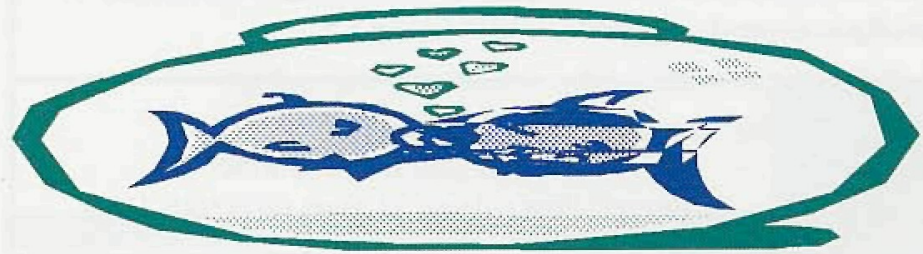

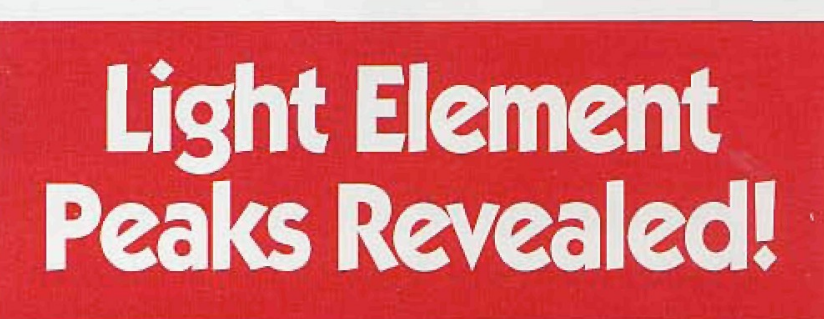

Oil Film on EDXWindows Removed:

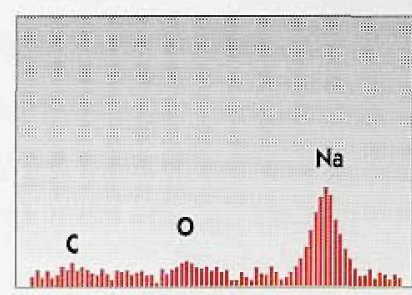

Oily Window

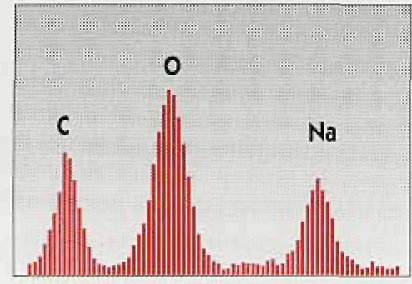

Clean Window
Oil build-up on EDX detector windows can ruin sensitivity for light element X-rays in SEMs. To stop oil condensation and keep the system clean, smart SEM users rely on the XEI Scientific SEM-CLEANTM system.

Result: Consistent light element X-ray results and contaminationfree pictures. The Nitrogen purge of the inexpensive SEM-CLEAN system actively cleans your electron microscope while you're away.

\section{SEM-CLEAN' Stops the Oil

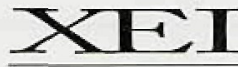 \\ SCIENTIFIC}

3124 Wessex Way, Redwood City, CA 94061 415-369-0133 - Fax 415-363-1659 